\title{
Children in England to get flu vaccine at age 2 years from September
}

Clarification-The new vaccination schedules mentioned in this News story are being introduced throughout the UK and not just in England (BMJ 2013;346:f2792, doi:10.1136/bmj. f2792). 